\title{
Training for Task: An Introduction to Part V, Training
}

\author{
Bill Fulford
}

With this penultimate part of the book, we come to the sharp end of values-based practice, namely the clinical skills that form the bedrock of the process on which it relies in supporting balanced decision-making on values in the context of clinical care. As described in chapter 1, "Surprised by Values: An Introduction to Valuesbased Practice and the Use of Personal Narratives in This Book", values-based practice builds on four main areas of learnable clinical skills, awareness, knowledge, reasoning, and communication skills. In this chapter, we fill out our descriptions of these skills areas and indicate how they are extended and enriched by the cultural values illustrated by the contributions to this Part (Table 33.1).

The training methods on which values-based practice has relied to date are derived largely from its original theoretical base in ordinary language philosophy. Part II, Theory, illustrated how the theory underpinning values-based practice could be much enriched by opening it up to other philosophical traditions, including those of non-Western cultures. The next two Parts explored aspects of this culturally enriched form of values-based practice, by way, respectively, of its model of service delivery (Part III, Practice) and of its role in linking science with people (Part IV, Science). The contributions to this Part explore aspects of this same cultural enrichment for the task of training for values-based practice. As we will see engaging with cultural values, while bringing with it new challenges for skills training, at the same time offers a much enriched resource base for the required training.

\footnotetext{
Authors

The editors with input from the contributors to Part V

B. Fulford $(\bowtie)$

St Catherine's College, University of Oxford, Oxford, UK

e-mail: kwmf@kwmfulford.com 
Table 33.1 Annotated Table of Contents for Part V, Training

Part V-Training

- Chapter 33 Training for Task: An Introduction to Part V, Training

V.1 Awareness of values

- Chapter 34 Values-Based Practice When Engaging with Voice-Hearers-Roz Austin

- Chapter 35 Dharma Therapy: A Buddhist Counselling Approach to Acknowledging and Enhancing Perspectives, Attitudes and Values-Sik Hin Hung and Jennifer Yim Shui Wa

V.2 Knowledge of values

- Chapter 36 Dangerous Liaisons: Science, Tradition, and Qur'anic Healing in the Dakhla Oasis of Egypt-Mohammed Rashed and Werdie van Staden

- Chapter 37 Know Thyself: Jane Discovers the Value of Her Depression-Tamara Kayali Browne

- Chapter 38 Case Studies in the Culture of Professional Football Players and Mental Welfare and Wellbeing-Michael Bennett

V.3 Reasoning about values

- Chapter 39 Sexual Orientation Change Efforts and VBP-Taryn Knox

- Chapter 40 Values, Meanings, Hermeneutics and Mental Health-Michael TH Wong

V.4 Communication skills for working with values

- Chapter 41 Disha: Building Bridges-Removing Barriers: Where Excluded and Privileged Young Adults Meet-Sadhana Natu

- Chapter 42 Online Counselling: The World Without a Label—Lejla Kuralić-Ćišić, Meliha Bijedić, Irma Dobrinjic, Nermina Kravić, Aida Duraković and Dajana Stajić

\subsection{Awareness of Values}

We start this Part with the first and foundational skills area for values-based practice, viz., raised awareness of values, and of the (often surprising) diversity of values.

The two brief exercises we included in chapter 1, "Surprised by Values: An Introduction to Values-Based Practice and the Use of Personal Narratives in This Book" - the 'three words exercise' and the 'forced choice exercise'-are illustrative of what has become the standard approach to awareness training in valuesbased practice. Developed originally by Kim Woodbridge-Dodd (the first author of chapter 44, "Reflections on the Impact of Mental Health Ward Staff Training in Race Equality and Values-Based Practice"), this was the approach adopted in the first training manual for values-based practice ('Whose Values?', [1]). Inspired by ordinary language philosophy $[2,3]$, the approach has subsequently been adopted across a range of clinical areas (see Guide to Further Information below). Powerful however as the approach has proved to be, that there remains much scope for improvement is evident, if in no other ways, from our own failure of self-awareness of values within values-based practice noted in chapter 1 (see section, 'Why now?').

The first two chapters in this Part illustrate the scope for further strengthening of values awareness within a culturally enriched form of values-based practice. Rosalind Austin (chapter 34, "Values-Based Practice When Engaging with VoiceHearers") illustrates the importance in this respect of personal narratives through the stories of two voice hearers, Paul and Mary. The two narratives, taken together, draw out an important feature of voice hearing that although neglected by services, is transparently self-evident within the cultural context of voice hearing. 
The neglected point in essence is this: that voice hearing although indeed sometimes a negatively evaluated experience (as it was for Mary) may sometimes come with distinct positive aspects as well (as it did for Paul). These positive aspects may as we have indicated be surprising to those (notably within services) for whom voice hearing is assumed to be a 'symptom' and hence necessarily a negative experience. But that this assumption of negativity may be clinically harmful is evident from Paul and Mary's stories. For as Rosalind goes on to show in her chapter, understanding the balance of positive and negative values attached to voice hearing for a given individual is vital to a recovery-oriented approach to clinical care.

The effectiveness of Rosalind and David Crepaz-Keay's work in the Educational Voice Hearers Network is one reason why in this book we based our approach so firmly on personal narratives. By allowing us to engage across and between cultures, personal narratives provide a powerful resource for establishing a culturally enriched form of values-based practice. Connecting with traditions in which the heuristic power of personal narratives has not been forgotten may facilitate this. In Part I, professional storyteller, Olusola Adebiye, with co-authors Tutiette Thomas and Temitope Ademosu, illustrated the healing power of storytelling in African culture with their chapter 11, on Madness, Mythopoetry and Medicine.

Chapter 35, "Dharma Therapy: A Buddhist Counselling Approach to Acknowledging and Enhancing Perspectives, Attitudes and Values" illustrates another such resource in this case represented by a Buddhist approach to mental health called Dharma Therapy. Mindfulness is of course nowadays familiar in Western mental health settings. But as Buddhist authors, Venerable Sik Hin Hung and Jennifer Yim, point out, mindfulness as it is currently practiced in Western mental health has, to its loss, become disconnected from its roots in Buddhist philosophy and practice. Their story of Peter Chan shows how Dharma therapy provides a more joined up approach. They emphasise that like any other therapy this is not for everyone. But where Dharma therapy works, an essential contribution to its effectiveness is the way that it helps to raise awareness of, and thus put those concerned in touch with, their true values, with what really matters in their lives.

\subsection{Knowledge of Values}

With the next three chapters, we move from awareness to knowledge of values. Before turning to individual contributions, there are two general points to bear in mind about knowledge of cultural values. The first is that-to put it bluntly-it is possible to get it. It is sometimes assumed that 'evidence of values' is somehow beyond the scope of empirical methods of enquiry. To the contrary, however, there are available a whole range of both qualitative and quantitative (and combined) methods available from the social and anthropological sciences for exploring the values of - for exploring what is important or matters to - this or that given group. We noted one such method in the introduction to Part III, Practice: the combined methodology adopted by Anthony Colombo and colleagues for exploring the 
implicit values of different professional groups within multidisciplinary mental health teams (chapter 15, "Vectors of Best Practice: An Introduction to Part III, Practice"). The contributions to this Part add further examples (see below).

With cultures, then, as with individuals, we should avoid just assuming that we know what is important to them. We have to, and we can, find out. But the second general point that is important to bear in mind about knowledge of cultural values is that such knowledge, however robustly derived, should never trump the values of a given individual. Recall the mantra about knowledge of values from chapter 1 that in values-based practice everyone is an ' $n$ of 1 '. Everyone, that is to say, however firmly embedded in his or her culture of origin, has a unique 'values fingerprint'. Knowledge of cultural values does have a proper role to play. It provides hints of or nudges towards what is likely to be important or matters to someone from a particular background or in a particular situation (a patient, for example, presenting with a given condition). But it is still important in any particular situation to find out what is in fact important or matters to the individual concerned. (We return to how this is done below, see 'communication skills').

Turning now to the contributions to this Part, three chapters illustrate in different ways how the resources of the social sciences can be harnessed to provide wellgrounded knowledge of cultural values. First, ethnography (chapter 36, "Dangerous Liaisons: Science, Tradition, and Qur'anic Healing in the Dakhla Oasis of Egypt") as used by Mohammed Rashid and Werdie van Staden in the former's study in the Dakhla oasis. Mohammed's deep immersion in the area over an extended period provides remarkable insights into the tensions of values between Western and nonWestern approaches to healing as these came together in the striking figure of a respected local Qur'anic healer, Sheikh Rayyes. The next chapter (chapter 37, "Know Thyself: Jane Discovers the Value of Her Depression"), based on its author's (Tamara K Browne's) study of people with depression, shows the power of the social sciences as reflected in an interview study. Echoing Rosalind Austin's work with voice hearers (chapter 34, "Values-Based Practice When Engaging with VoiceHearers") Tamara shows through the story of one of her participants how depression, although for most people a deeply negative experience, may nonetheless come with positive aspects. There are pointers to this in historical work on depression (or its cognates, [4]). But in contemporary contexts, as Tamara's work illustrates, it is from the social sciences that we are able to derive fine-grained and reliable information as the basis of person-centred clinical care.

With the third chapter on knowledge, Michael Bennet's (chapter 38, "Case Studies in the Culture of Professional Football Players and Mental Welfare and Wellbeing") work with professional footballers, we come to a novel and positive resolution of the tension between individual and cultural values. Michael's study uses discourse analysis to explore the findings from his interviews with a number of players from different clubs in the UK professional football league. In this chapter, he describes one of a number of emerging themes, called by one of his interviewees 'The Snowball Effect'. 
So described, the study, although presenting novel and important findings, may appear unremarkable as an application of social science methods. But the study is innovative at a number of levels. It is the first in-depth qualitative study of what matters or is important to professional footballers. There have been many rather superficial quantitative studies. But Michael's is the first to dig below the surface to provide well-grounded understanding of the issues. It is also the first such study to be carried out with clinical interventions explicitly in mind-as a qualified therapist and Head of Welfare for the game's governing body in the UK, the PFA (Professional Footballers Association), Michael was motivated in part by the fact that to date professional football has been a neglected area of mental health. And, Michael being himself an ex-professional footballer, it is the first such study to combine rigorous social science methods with personal experience of the field of study.

It is in this third respect, in the combination his study offers of rigorous methods with personal experience, that Michael provides a resolution of the tension between individual and cultural values. His approach is highly rigorous methodologically. But as an ex-professional footballer himself, his approach is not that merely of a detached researcher. There are of course methodological pitfalls in adopting an engaged rather than detached perspective in work of this kind. But there are methodological pitfalls too in being detached. This is why combining his personal experience with the use of a rigorous social science method makes Michael's study so important. It means that he brings a unique combination of expertise by training and expertise by experience to his work. The result is a rigorous qualitative social science study of the culture of professional football that is informed and filled out by Michael's personal experience as a former professional footballer. Uniquely illuminating, it provides insights into the values of people working within a culture to which most of us have either no access from personal experience or, worse, the potentially misleading access provided by media coverage. In Michael's hands, moreover, the study provides these crucial insights explicitly in the service of guiding therapeutic interventions.

\subsection{Reasoning About Values}

Reasoning about values has a long tradition in moral philosophy on which contemporary bioethics has drawn freely. Values-based practice, too, has drawn on the same traditions of moral reasoning but with an important difference. Where bioethics has (in the main) used the traditions of moral reasoning as a way of getting 'right answers', values-based practice has drawn on these traditions with the more modest aim of broadening understanding of the values in play in the situation in question.

The difference we should say straight away is not as stark as we have drawn it here. In early editions, for example, of the seminal Four Principles of Biomedical Ethics, its authors (the philosopher Tom Beauchamp and the theologian James Childress) emphasised that their eponymous four principles were intended to 
provide a framework, no more and no less, for mapping out the dimensions of an ethical issue in medicine [5]. This is exactly how 'principles reasoning' is used in values-based practice, to map out the values in play. For example, a values-based approach to the use of mental health legislation in the UK used principles reasoning in this 'mapping out' way [6]. As in bioethics again, so also in values-based practice, the top-down approach of principles reasoning has to be balanced with the bottom-up approach of case-based reasoning (or casuistry). The latter connects with our point in chapter 1 , about the power of narrative to illuminate values. Philosophers, Albert Jonsen and Stephen Toulmin, reintroducing casuistry to medical ethics [7], noted how the appeal to concrete case examples tapped into our implicit understanding of a situation.

Values-based practice, in drawing on traditional forms of moral reasoning in this 'mapping out' way, connects with other approaches in the 'values tool kit'. It follows, for example, the precedent set by Oxford's first Professor of Medical Ethics, Tony Hope, working with Bill Fulford, in the Oxford Practice Skills Project [8]. This project established the general approach of partnership between ethics/values and science in clinical decision-making that now underpins values-based practice. As a teaching resource for medical students, it brought together for the first time, ethics, law, and communication skills with evidence-based medicine as the basis of clinical decision-making. Values-based practice also has parallels with contemporary movements in bioethics towards developing practical resources for problemsolving (as distinct from prescriptive ethics). These movements are called variously 'clinical ethics' or 'practical ethics' (see, for example, [9]).

In this Part, we have included two chapters that between them illustrate the resources of traditional forms of moral reasoning applied to cultural values. Taryn Knox' detailed case study (chapter 39, "Sexual Orientation Change Efforts and VBP") makes the point for case-based reasoning in her powerful explication of the conflicting cultural and other values involved in decisions about conversion therapy for homosexuality. The story in this chapter shows just how sensitive such decisions are to the specific contingencies presented by the particular situation. The balance of considerations in her target case as Taryn points out, might have come out very differently if, say, contrary to current realities, there were a form of conversion therapy available of proven safety and effectiveness.

Case-based reasoning may be used to good effect in isolation. Combining it with other methods can however boost its powers of explication. One such method, as Michael Wong, from the University of Hong Kong, illustrates in chapter 40, "Values, Meanings, Hermeneutics and Mental Health", is hermeneutic analysis. His narrative case study is of 'Mary', a woman in her middle years, who is referred to the local Neuropsychiatry Clinic for assessment of persistent abnormal movements believed to be secondary to the medication she had been prescribed for a pre-existing condition (depression with psychotic features). Her management followed standard medical protocols. But these proved effective only when combined with hermeneutic analysis used to deepen understanding of the cultural and other values driving her medical presentation. 


\subsection{Communication Skills}

Values-based practice as has been said is 'nothing without communication skills' ([10], chapter 7). Communication skills are important to values-based practice in two specific respects: for eliciting values (recall the listening skills shown by the surgeon in the story of Mrs. Jones' knee in chapter 1, "Surprised by Values: An Introduction to Values-Based Practice and the Use of Personal Narratives in This Book") and for conflict resolution.

As with other areas of values-based practice, its relationship with communication skills is that of a two-way partnership. Communication skills contribute in these two specific (and other general) ways to values-based practice. Values-based practice in turn contributes content to communication skills. The values-based concept of StAR values (Strengths, Aspirations, and Resources), as used in teaching, for example, is combined with the ICE (Ideas, Concerns, and Expectations) of standard communication skills training, to give the mnemonic ICEStAR. This reminds students to explore positives (StAR values) as well as negatives (ICE) with their patients.

The final two chapters in this Part show the potential contributions of culturally enriched forms of values-based practice for both aspects of values-based communication skills: for eliciting values (chapter 41, "Disha: Building Bridges-Removing Barriers: Where Excluded and Privileged Young Adults Meet") and for conflict resolution (chapter 42, "Online Counseling 'the World Without a Label"'). Reporting from Pune in India, Sadhana Natu (chapter 41) describes their innovative Disha programme. Through case studies and personal testimonies, she illustrates how bringing students together from diverse backgrounds within peer support groups provides a powerful way for participants to become more aware of their own and others' values. The Disha programme is thus a model for the 'open society of stakeholders' that, as we describe in our concluding chapter (chapter 47, "Co-Writing Values: What We Did and Why We Did It") and After Word, it is the ultimate aim of this book to establish.

Chapter 42, "Online Counseling 'the World Without a Label'”, by Irma Dobrinjic and colleagues, from the University of Tuzla in Bosnia and Herzegovina, illustrates the potential of new technology in support of the communication skills for conflict resolution in values-based practice. Conflict resolution is well developed for faceto-face encounters. But coming face-to-face is precisely what the conflicts in Irma Dobrinjic and colleagues' group of clients (adolescents and their families) are all about. Through the story of Adnan and his family, they show how effective their online counselling service may be in helping to manage the conflicts of values arising between young people with behavioural problems and their parents over engagement with services. The young people in question, as their case narrative illustrates, are brought to services by their parents but reject help for fear of being stuck with a label. Computer literate however as these young people invariably are, they engage readily and often to good effect when counselling is offered in an online form. Such counselling is available at a time of the young person's choosing and being anonymous it comes literally, as their title has it, 'without a label'. 
As with Sadhana Natu's Disha programme, there are lessons from Irma Dobrinjic and colleagues' online counselling service for the "open society of stakeholders" that, as we describe in our concluding chapter (Chapter 47) and After Word, it is the ultimate aim of this book to establish. They indeed comment on the potential for developing similar online counselling services in other situations around the world. As an exercise in conflict resolution, the effectiveness of their approach is all the more impressive for the fact that it has been developed in the challenging post-war circumstances currently obtaining in Bosnia and Herzegovina.

\subsection{Conclusions}

This Part of the book is concerned with the training implications for a culturally enriched form of values-based practice. In each of the areas covered-awareness, knowledge, reasoning, and communication skills-the implications cut both ways. Engaging with cultural values raises new challenges for training in the skills for values-based practice. But such engagement also offers new assets for and models of training.

The communication skills for values-based practice, the topic of the last two chapters in the Part, provide particularly powerful illustrations of this two-way cut. Sadhana Natu's Disha programme in Pune (chapter 41, "Disha: Building BridgesRemoving Barriers: Where Excluded and Privileged Young Adults Meet") and Irma Dobrinjic and colleagues' online counselling service in Tuzla, Bosnia and Herzegovina (chapter 42, "Online Counseling 'the World Without a Label'"), have both been developed in challenging local circumstances, respectively, extreme economic and social differences among students, and post-war conflict. Yet both have developed effective ways of delivering culturally appropriate values-based mental health services. Their examples thus show that challenging as training for the task of delivering a culturally enriched form of values-based practice may be, it is a task that can nonetheless be done.

\subsection{Guide to Further Information}

For more on skills training in values-based practice, please see the website for the Collaborating Centre for Values-based Practice in Oxford at: valuesbasedpractice. org/More about VBP.

This includes a number of exemplar training materials that are available as free full-text downloads.

\section{References}

1. Woodbridge K, Fulford KWM. Whose values? In: A workbook for values-based practice in mental health care. London: The Sainsbury Centre for Mental Health; 2004.

2. Fulford KWM. Moral theory and medical practice. Cambridge: Cambridge University Press; 1989. (Reprinted 1995 and 1999). 
3. Fulford KWM. Living with uncertainty: a first-person-plural response to eleven commentaries on values-based practice, chapter 13. In: Loughlin M, editor. Debates in values-based practice: arguments for and against. Cambridge: Cambridge University Press; 2014.

4. Fulford, KWM, Crepaz-Keay D, Stanghellini, G. Depressions Plural: pathology and the challenge of values. Chapter 14, pps 141-158 in charles foster and jonathan herring (eds) depression: law and ethics. Oxford: Oxford University Press

5. Beauchamp TL, Childress JF. Principles of biomedical ethics. 3rd ed. Oxford: Oxford University Press; 1989.

6. Fulford KWM, Dewey S, King M. Values-based involuntary seclusion and treatment: value pluralism and the UK's Mental Health Act 2007. Chap. 60. In: Sadler JZ, van Staden W, Fulford KWM, editors. The oxford handbook of psychiatric ethics. Oxford: Oxford University Press; 2015.

7. Jonsen AR, Toulmin S. The abuse of casuistry: a history of moral reasoning. California: University of California Press; 1988.

8. Hope T, Fulford KWM, Yates A. The Oxford practice skills course: ethics, law and communication skills in health care education. Oxford: The Oxford University Press; 1996.

9. Persson I, Savulescu J. Unfit for the future: the need for moral enhancement (Uehiro series in practical ethics). Oxford: Oxford University Press; 2012.

10. Fulford KWM, Peile E, Carroll H. Essential values-based practice: clinical stories linking science with people. Cambridge: Cambridge University Press; 2012; Japanese edition, Medical Sciences International 2016; French edition, John Libbey Eurotext, 2017.

Open Access This chapter is licensed under the terms of the Creative Commons Attribution 4.0 International License (http://creativecommons.org/licenses/by/4.0/), which permits use, sharing, adaptation, distribution and reproduction in any medium or format, as long as you give appropriate credit to the original author(s) and the source, provide a link to the Creative Commons license and indicate if changes were made.

The images or other third party material in this chapter are included in the chapter's Creative Commons license, unless indicated otherwise in a credit line to the material. If material is not included in the chapter's Creative Commons license and your intended use is not permitted by statutory regulation or exceeds the permitted use, you will need to obtain permission directly from the copyright holder.

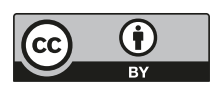

